The daylight sky and Avogadro's number

This content has been downloaded from IOPscience. Please scroll down to see the full text. 2015 Eur. J. Phys. 36065040

(http://iopscience.iop.org/0143-0807/36/6/065040)

View the table of contents for this issue, or go to the journal homepage for more

Download details:

IP Address: 159.149.197.10

This content was downloaded on 23/03/2016 at $11: 20$

Please note that terms and conditions apply. 


\title{
The daylight sky and Avogadro's number
}

\author{
Marco A C Potenza \\ Department of Physics, via Celoria 16, I-20133 Milan, Italy \\ E-mail: marco.potenza@unimi.it
}

Received 17 February 2015, revised 25 June 2015

Accepted for publication 27 July 2015

Published 6 October 2015

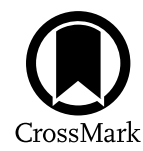

\begin{abstract}
Two methods for estimating Avogadro's number from the observation of the daylight sky are presented, both suitable for undergraduate students. One is very simple and based on simple naked-eye observation, and the other exploits a common digital camera as a photometer.
\end{abstract}

Keywords: light scattering, fundamental constants, photometry

(Some figures may appear in colour only in the online journal)

\section{Introduction}

Every amateur astronomer knows that the Sun, the Moon and the planet Venus are the only celestial bodies visible in the daylight, Venus being close to the limit of visibility for the naked eye. Under a dry and unpolluted sky, preferably near maximum elongation from the Sun, Venus can be observed at noon, with the Sun very high over the horizon. Here we show how this actually gives enough information for a rough estimate of Avogadro's number $\left(N_{\mathrm{A}}\right)$. The origin of daylight, one of the earliest question of philosophers and scientists wondered about, is ultimately related just to the molecular composition of the atmosphere. Providing evidence for the actual existence of atoms and molecules was a challenge for scientists at the beginning of the twentieth century, when people like Maxwell, Perrin, and Einstein, among others, devised a number of methods for measuring $N_{\mathrm{A}}$. In 1899 Rayleigh estimated the amount of light scattered by air molecules and related it to the scattering of sunlight. In 1910 Einstein, following Smoluchowski [1], found a formula for evaluating the intensity of light scattered by density fluctuations in a fluid [2], thus giving an unexpected solution to many problems that the simple question of the origin of daylight had led to. The sky is still far from completely understood [3, 4], but the milestone work done by Rayleigh and Einstein leads to an explanation complete enough for a quantitative description of daylight.

In this work I first introduce the issue of daylight and the most fundamental questions animating the dispute a century ago (section 2). Both Einstein's and Rayleigh's approaches are presented to derive an expression for the brightness of the sky (sections 3 and 4). To 
estimate $N_{\mathrm{A}}$, two methods are presented: 1 ) from the daylight observation of the planet Venus, which is very easy but only gives a lower limit (section 5), and 2) from digital pictures of the sky taken with a digital camera, properly calibrated (section 6). Both methods and the necessary mathematics are simple enough to make it a suitable experiment for undergraduate students, as I have experienced several times in recent years.

\section{The puzzle of the blueness of the sky}

Before Einstein, the early contributors to the explanation of the sky's blueness were Goethe, Tyndall and Rayleigh, who introduced the scattering of light by small particles (Goethe's urphaenomenon [3]), studied the diffusion of light from a dilute suspension (Tyndall light) [5] and described the light scattered from a point-like oscillator (Rayleigh scattering) [6]. After a suggestion by Maxwell, Rayleigh applied his own theory to the scattering of light from air molecules [7]. He thus related $\mathrm{N}_{\mathrm{A}}$ to the so-called visual range, the maximum distance at which an object can be seen through pure air [8] (thus also giving an explanation for the aerial perspective used by Leonardo da Vinci in painting far objects). The result is in rough agreement with the accepted value for $N_{\mathrm{A}}$.

Rayleigh opened a new era in the study of daylight, overcoming the main problem of Goethe and Tyndall's approaches. Air molecules are always there, while dust depends on several conditions: what would happen without dust? With Rayleigh's theory the answer is in the atmosphere itself.

Nevertheless, Rayleigh used an important assumption that is worth discussing, as it captured the attention of remarkable thinkers a century ago, driving a scientific controversy that is almost forgotten nowadays. He considered the scattering by air molecules to be incoherent, meaning that the intensity of light perceived by an observer looking towards an ensemble of molecules is just the sum of the intensities of the light scattered by each molecule (as if each was isolated). This actually occurs when the phases of the scattered waves are random, for example due to the random positions of rarefied random scatterers. But air is not so rarefied as to fit this assumption, since the average distance between molecules at sea level is only $3 \mathrm{~nm}$, much smaller than the wavelength of visible light (on the order of half a micron). A huge number of molecules are driven in phase by the incoming field within a distance comparable to the wavelength. For each molecule scattering light with a given phase, another molecule half a wavelength away will scatter light with the opposite phase, thus producing destructive interference. The only constructive interference will be in the forward direction (coherent scattering), where the incoming wave is simply phase lagged, giving the air its refractive index. For this reason Mandelstam [9] was convinced that the Rayleigh theory was wrong, since it leads to the conclusion that air must be completely transparent, against observation. By contrast, Rayleigh (and Planck) argued that the scattering should be considered incoherent due to the thermal motions of the molecules, which randomize the phases (even if they did not explain how). Smoluchowski [1] and Einstein [2] entered this controversy from a different point of view, the study of the scattering by density fluctuations in fluids. Einstein gave a completely new description that leads to a result formally identical to that of Rayleigh (as Einstein himself noted in his paper; for a proof of this equivalence see for example [10]). With no fluctuations at all, light would pass undisturbed through the medium, as Mandelstam correctly pretended. But the air is not really uniform, and small density fluctuations locally produce different scattering efficiencies, affecting the coherence phenomena responsible for constructive interference in the forward direction and destructive interference in any other direction. Incidentally, Einstein noticed that the direct assumption of 
the atomic structure of matter is not really necessary in his theory, since he modeled the fluid as a continuum. Nevertheless, he proved that the atomic structure is of fundamental importance from a statistical point of view, in determining the density fluctuations within the fluid.

If molecules move independently, as they do in air at standard temperature and pressure (STP), the number of molecules within a given volume will change accordingly to Poisson's statistics: the fluctuations will depend on the number of molecules. On average, coherent scattering by the fluctuations of $N$ molecules gives the same intensity as incoherent Rayleigh scattering by the same $N$ molecules. In the former case the observed intensity is the sum of $\sqrt{ } N$ field contributions, while in the latter it is given by the sum of $\mathrm{N}$ equal-intensity contributions [11, 12]. If fluctuations are not driven by Poisson's law, as occurs for example in liquids, incoherent Rayleigh scattering will not give the correct description of the phenomenon.

It can also be noticed that the size of the density fluctuations we are dealing with must be much smaller than the wavelength, since daylight is isotropically scattered.

A question still remains: in usual conditions, is daylight due to air or dust? The Rayleigh scattering law is the same for both cases, as for any other object much smaller than the wavelength. The blue coming from air is identical to that coming from dust particles. A spectral measurement cannot distinguish between the two cases: only the scattering efficiency changes, this change being with the sixth power of the size.

\section{Einstein's equation}

Einstein [2] related the intensity $I$ of the light scattered by a gas to the intensity of the impinging light, $I_{0}$ :

$$
\frac{I}{I_{0}}=\frac{R T}{N_{A}} \frac{(\varepsilon-1)^{2}}{p}\left(\frac{2 \pi}{\lambda}\right)^{4} \frac{\Phi}{(4 \pi D)^{2}} \sin ^{2} \phi
$$

where $R$ is the gas constant, $T$ the absolute temperature, $\varepsilon$ the dielectric constant of the gas, $p$ the pressure, $\Phi$ the illuminated volume, $D$ the distance between the volume and the observer, and $\phi$ the angle between the polarization of the incoming light and the scattering plane (the plane containing both the incoming and the emerging beams).

By integrating over the solid angle the intensity of light scattered with any polarization state, substituting $(\varepsilon-1)^{2}=4(n-1)^{2}\left(n^{2}=\varepsilon \sim 1\right.$ for air at STP and at optical frequencies), and $R T / p$ with $N_{\mathrm{A}} / N$ from the perfect gas law (the number density is $N=\rho N_{\mathrm{A}} / M$, with $\rho$ the gas density and $M$ the molecular weight of air), one obtains the scattered power:

$$
\frac{P}{I_{0}}=\frac{2}{3 \pi} \frac{M}{N_{A}} k^{4} \frac{(n-1)^{2}}{\rho} \Phi
$$

where typically $k=2 \pi / \lambda=1.3910^{7} \mathrm{~m}^{-1}$ for blue light $(\lambda=450 \mathrm{~nm})$ and $M=28.96 \mathrm{~g} \mathrm{~mol}^{-1}$ for air.

Here we refer to a simplified model of the atmosphere based on the isothermal assumption, which despite its simplicity gives a good approximation of the real case, as detailed for example in [13]. Therefore, gas density $\rho$ decreases with altitude $z$ with an exponential law $\rho=\rho_{0} \exp \left[-z / z_{0}\right]$, with $\rho_{0}=1.210^{-3} \mathrm{~g} \mathrm{~cm}^{-3}$ and $z_{0}=8800 \mathrm{~m}$ (see for example [8]). In order to evaluate the term $(n-1)$ and its dependence on the altitude $z$, recall the so-called Lorentz-Lorenz (LL) formula [14], relating the polarizability of the single molecule, $\alpha$, to the refractive index $n$ of a gas composed of the same molecules with number density $N$ : 


$$
3 \frac{n^{2}-1}{n^{2}+2}=4 \pi \alpha N
$$

This result holds true if $N$ is large enough that a large number of molecules are present within a volume comparable to a cube one wavelength on a side $[15,16]$. The term $(n-1)=\left(n^{2}-1\right) / 2$ is then proportional to $N$, and therefore to the gas density $\rho$. The term $(n-1)$ will then be described by $(n-1)=\left(n_{0}-1\right) \exp \left[-z / z_{0}\right]$, and $(n-1)^{2} / \rho$ can be simplified to $\left(n_{0}-1\right) / \rho_{0} \exp \left[-z / z_{0}\right]$. For air at STP conditikns, the refractive index is given by the Cauchy relation:

$$
n_{0}=A+\frac{B}{\lambda^{2}}+\frac{C}{\lambda^{4}}
$$

with $A=1.000287566, B=1.341210^{-18} \mathrm{~m}^{2}$, and $C=3.77710^{-32} \mathrm{~m}^{4}$. For blue light at $\lambda=450 \mathrm{~nm}$ this gives $n_{0}=1.00029$, or $n_{0}-1=2.9 \times 10^{-4}$.

Since the sky is an extended source we will consider its photometric radiance, namely the power received from a unit solid angle $\Omega$ on a unit surface on the ground. For daylight coming from the zenith, such that $D=z$, the volume $\mathrm{d} \Phi$ contributing to the light impinging on the ground from height $z$ will be determined by the reference solid angle $\Omega, \mathrm{d} \Phi=\Omega z^{2} \mathrm{dz}$. Also, we will refer to a unit surface $S$ on the ground, determining a solid angle $\omega(z)=S /\left(4 \pi z^{2}\right)$ at height $z$. The radiance of the daylight at ground level will then be obtained by integrating over the entire air volume determined by the solid angle $\Omega$. We will assume that the solar irradiance $I_{0}$ (power per unit surface, here considered in the blue range of the solar spectrum) is uniform over the entire height of the atmosphere, which for blue light and pure air is correct within $20 \%$ (as is evident from a comparison of the extraterrestrial and the sea level spectral irradiances). This gives

$B=\frac{I_{0}}{S \Omega} \int_{0}^{\infty} d z \frac{2}{3 \pi} \frac{M}{N_{A}} k^{4} \frac{\left(n_{0}-1\right)^{2}}{\rho_{0}} \exp \left[-z / z_{0}\right] \frac{\Omega S}{4 \pi}=I_{0} \frac{2}{3 \pi} \frac{M}{N_{A}} k^{4} \frac{\left(n_{0}-1\right)^{2}}{\rho_{0}} z_{0} \frac{1}{4 \pi}$

which can be inverted to obtain the value for $N_{\mathrm{A}}$ once $B$ and $I_{0}$ are known. The result can be easily generalized for the intensity of daylight observed from any altitude $z_{\text {obs }}$.

\section{Rayleigh scattering}

Here we will briefly address the same issue following Rayleigh, since this is the most common way the result is derived in the literature. For a single, spherical dielectric particle much smaller than the wavelength $(<\lambda / 20)$ of polarizability $\alpha$, the Rayleigh cross section is given by [5]:

$$
\sigma_{S}=\frac{8 \pi}{3} k^{4}|\alpha|^{2}
$$

To evaluate the polarizability $\alpha$ of each molecule we will use the LL formula (3) again, with the approximations $n_{0}{ }^{2}+2=3$ and $n_{0}{ }^{2}-1=2\left(n_{0}-1\right)$, and get the rough estimate $\sigma_{S}=10^{-30} \mathrm{~m}^{2}$.

Alternatively, one can obtain a similar result on the basis of the most elementary model for the polarizability, that is, by considering the resonances of electric dipoles with proper frequencies $\omega_{k}(\omega=2 \pi c / \lambda$ ( $c$ is the speed of light in vacuum)). The polarizability is given by (see for example [17]): 


$$
\alpha=\frac{e^{2}}{m_{\mathrm{e}}} \sum_{k} \frac{f_{k}}{\omega_{k}^{2}-\omega^{2}}
$$

where $e$ and $m_{\mathrm{e}}$ are the electron charge and mass, and $f_{k}$ are the oscillator strengths for the resonances at frequencies $\omega_{k}$. The strongest emission for nitrogen molecules occurs at $\lambda_{0}=120 \mathrm{~nm}$, the Lyman $\alpha$ of the $\mathrm{N}_{2}$ molecule, which is also the strongest line in the atmosphere [18, 19]. The polarizability can be approximated as due to one oscillator with $f_{k}=1$, and for $\lambda=450 \mathrm{~nm}$ one finds $\omega=0.27 \omega_{0}$. Since $e^{2} / m_{\mathrm{e}}=c^{2} r_{0}$ $\left(r_{0}=2.82 \times 10^{-15} \mathrm{~m}\right.$ is the classical electron radius), it is easy to find a value for the cross section that gives results just slightly smaller than the one evaluated above, namely $\sigma_{S}=4 \times 10^{-31} \mathrm{~m}^{2}$. For the sake of completeness, we note that the radiation damping for atomic transitions lasts for something like $10^{-8} \mathrm{~s}$, so the linewidth is of the order of $10^{8} \mathrm{~Hz}$, which is negligible with respect to the difference between the frequencies $\omega_{k}$ and $\omega$. We conclude that the approximation used to write equation (7) does not introduce appreciable uncertainties.

Nitrogen is actually a diatomic molecule, with an anisotropic charge distribution that alters the pure Rayleigh scattering. Nevertheless, on the basis of the results obtained by King [20], this would affect the polarizability by a factor $F(\omega)=1.034+0.080 \omega^{2}$. For blue light this amounts to about $F=1.036$, so it is negligible here.

The incoherent scattered intensity coming from $N$ molecules is simply $\sigma_{S} N$. Dividing for a given volume, we get a specific scattered intensity equal to $\sigma_{S} N$. Given the density profile $\rho(z)$ above, we will immediately get the number density profile in terms of $N_{\mathrm{A}}, N(z)=N_{0} \exp$ $\left[-z / z_{0}\right]$, where $N_{0}=\rho_{0} N_{\mathrm{A}} M^{-1}$.

The radiance at the ground of daylight from the zenith will therefore be given by:

$$
B=I_{0} \int_{0}^{\infty} \mathrm{d} z \sigma_{S} N_{0} \exp \left[-z / z_{0}\right] \omega(z) z^{2}
$$

or:

$$
B=I_{0} \sigma_{S} N_{0} z_{0} / 4 \pi
$$

which can be easily reduced to equation (5). This expression is particularly suitable for finding the radiance of the daylight if $N_{\mathrm{A}}$ is known.

\section{Estimating Avogadro's number from observations of Venus}

The sky's brilliance can be estimated on the basis of two simple observations: (1) Venus' light is close to the limit of naked eye visibility over daylight from a very clear atmosphere; and (2) the angular resolution for the naked eye adapted for diurnal vision of the bright sky is approximately $\theta=80-100$ arcsec, very close to the apparent diameter of Venus, 80-90 arcsec. This allows us to derive a limit to the specific magnitude of the sky.

Astronomers express the intensities of light in units of magnitude, defined as follows:

$$
m=-2.5 \log \left(I / I^{*}\right)
$$

where $I$ is the intensity of light coming from a celestial object, as measured in a reference spectral range, and $I^{*}$ is a standard intensity in the same spectral range (conventionally considered to be the intensity of light coming from Vega in the visible range, that is, $1.2 \times 10^{-8} \mathrm{~W} \mathrm{~m}^{-2}$ ). The magnitude of Venus is approximately -4 and almost constant (with a minimum of -4.6); this value gives an upper limit for the specific magnitude of the sky as about $-3 \operatorname{arcmin}^{-2}$. Since the Sun has a magnitude of -26.72 and an irradiance of about $600 \mathrm{~W} \mathrm{~m}^{-2}$ at sea level in the visible range, we can easily estimate the intensity of light 
coming from the sky. The last needed piece of information comes from the spectral response of the human eye; response in the blue is approximately $1 / 10$ that in the yellow-green [22], where the light from Venus will mainly be sensed. Therefore, we estimate $I=3.0-7.5 \times 10^{-7} \mathrm{~W} \mathrm{~m}^{-2}$.

Using equation (5) and the numerical values evaluated above $(M=28.96$, $k=1.39 \times 10^{7}, \quad\left(n_{0}-1\right)^{2}=8.4 \times 10^{-8}, \quad \rho_{0}=1.2 \times 10^{3} \mathrm{~g} \mathrm{~m}^{-3}, \quad z_{0}=8800 \mathrm{~m}$, $\Omega=1.1 \times 10^{-6} \mathrm{Sr}$ ), and since the solar irradiance in the blue range is approximately $I_{0}=180 \mathrm{~W} \mathrm{~m}^{-2}$ [23], one obtains a lower limit for $N_{\mathrm{A}}$ :

$$
N_{A}>4.6 \times 10^{23}
$$

the correct order of magnitude. The main source of error here can be ascribed to the dust suspension in the atmosphere, which increases the intensity of daylight with respect to that of pure air, and leads us to underestimate $N_{\mathrm{A}}$ (see equation (5)).

\section{Measuring Avogadro's number through a digital camera}

The advent of digital cameras and webcams allowed us to easily handle quantitative data by converting pictures into arrays of numbers. Advanced data reduction schemes are commonly used by amateur astronomers.

Here the use of commercial cameras as absolute photometers is proposed to measure the sky radiance. Any camera can be used, provided that direct control of the objective settings (focal length and diaphragm aperture) and white balance is possible. Otherwise there is no chance to calibrate the camera's response. Commercial cameras that produce any kind of RGB pictures in standard formats (TIFF, JPG, BMP, etc) are suitable for this purpose. The case of RAW images produced by professional cameras is not considered here. Several cameras have been tested successfully, with similar results. The following steps will be described: (1) setting the conditions to be used in the measurements; (2) measuring the response curve of the camera; (3) determining the absolute calibration with a known source; and (4) collecting and reducing data from the daylight sky.

\subsection{Measurement conditions}

The camera objective, focal length, f-number, must be fixed. Particular care has to be taken to prevent any firmware/software data processing by the camera before the data is stored. For the data reported below, the camera was a Nikon D70 set to manual configuration, ISO 200, $\mathrm{f} / 4.5,70 \mathrm{~mm}$ focal length.

\subsection{Calibration of the response curve}

Digital cameras ultimately convert light intensity (photons) into electrons generated within the potential wells associated with each pixel. This conversion is usually linear, so that the number of electrons is proportional to the energy collected, namely the number of photons. Pictures are generated by the firmware of the camera by introducing a nonlinear response, which mimics the response of traditional film emulsions. Inverting this function is the aim here, in order to recover the intensity of light. The calibration of the response function can be easily measured through the following procedure (see [24] for a detailed description of the calibration procedure). (1) Preparing a uniformly illuminated plane surface: the clear sky close to the zenith is particularly suitable, provided that the overall measurement is fast enough that the daylight will not change while pictures are taken (this is the case considered 


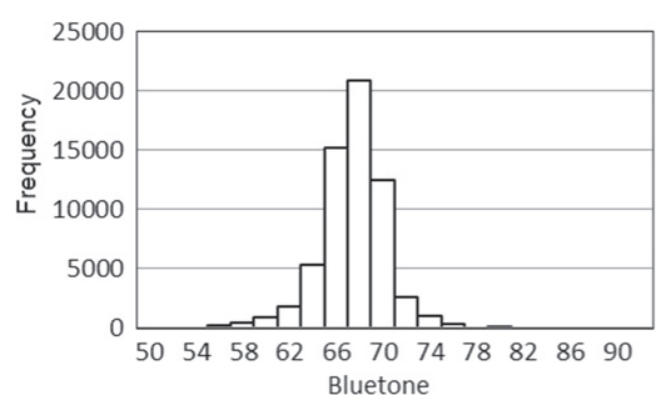

Figure 1. Example of frequency distribution $n(b)$ for data used in this work.

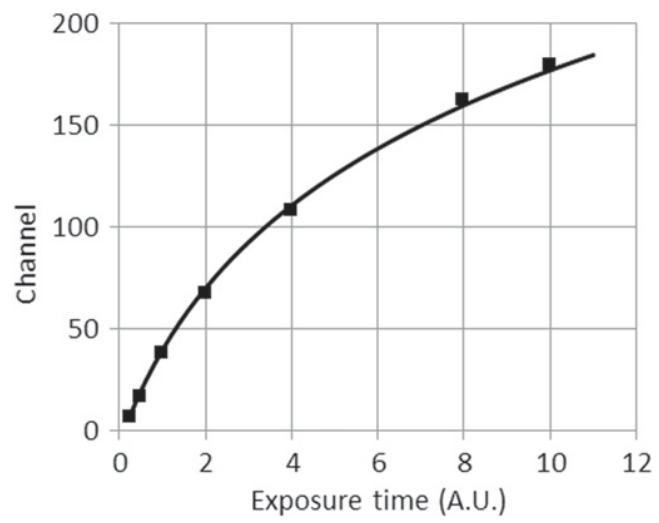

Figure 2. Response curve as measured by the procedure described in the text. The $x$ axis shows the exposure times (arbitrary units), and the $y$-axis shows the corresponding average tones of the frequency histograms.

here). (2) Taking a set of pictures with increasing exposure times, starting from a picture almost completely dark and progressing to a picture completely saturated in the blue channel. (3) Importing images to a PC and measuring the frequency histogram of the blue channel, which provides the number $n$ of pixels assigned to a given tone, $b$, which ranges from 1 to 256 for a typical 8-bit color coding. Notice that the tone $b$ is not the number of electrons collected by each pixel, a further constant being here that is related to the sensitivity of the camera (ISO setting). The central region of the picture is preferable, in order to exclude unavoidable vignetting effects.

This distribution must be very narrow, which indicates that daylight is really uniform over the image (see figure 1). By extracting the average tone from each picture and plotting the values as a function of the corresponding exposure time $\Delta t$, the response curve is obtained. In figure 2 the experimental results are plotted as a function of arbitrary units on the $x$-axis, ultimately proportional to the number of electrons collected by each pixel.

The curve can easily be fitted with typical empirical formulas. In the case of figure 2 we get:

$$
b(c)=90 \log _{10}(10 c+15)-250
$$




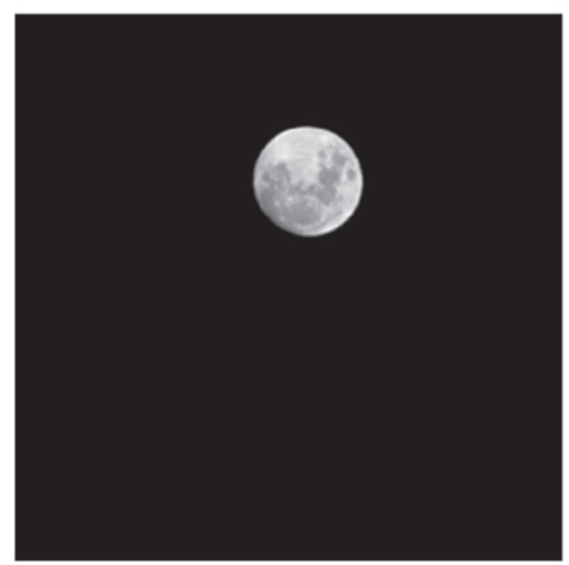

(a)

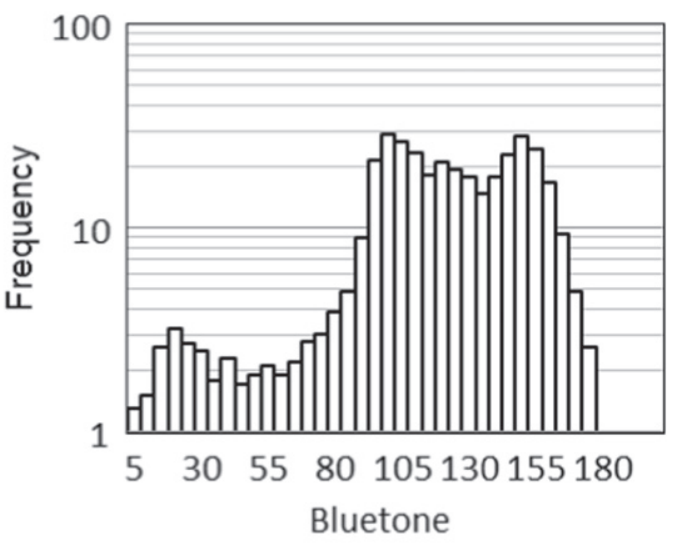

(b)

Figure 3. (a) An example of the pictures used for absolute calibration; (b) the corresponding frequency histogram ( $x$-axis bluetones, $y$-axis frequencies plotted on a $\log$ scale).

where $c$ is the arbitrary counts unit. By inverting:

$$
c(b)=(\exp [(b+250) / 90]-15) / 10
$$

This can be considered accurate enough from tones 20 to 175, which will be enough for our purposes. Notice that $c(b)$ is ultimately related to the energy emitted from a given solid angle of the sky, falling on a given area on the ground within the exposure time $\Delta t$ through the objective with numerical aperture $f$. The solid angle is subtended by the projection of each pixel onto the sky through the camera objective, and the unit surface is the pixel area.

Therefore, by normalizing $c(b)$ for the exposure time $\Delta t$ and the diaphragm aperture, that is the inverse of the $\mathrm{f}$ number squared, a quantity is obtained that is proportional to the power emitted within a unit solid angle, impinging on a unit area at ground level, namely to the radiance:

$$
A(b)=c(b) f^{2} / T
$$

$A(b)$ will be referred to as the relative radiance. It is worth noticing that relation (11) could introduce some discrepancies when measuring $A(b)$ for different $f$ values. To get the best results, this parameter should be kept fixed.

\subsection{Absolute photometry}

Here the constant relating the relative radiance $A(b)$ to the absolute radiance is determined. This requires a known source. The moon is suitable, since it is an extended source and allows calibrating the energy collected by each pixel coming from the corresponding solid angle. Moreover, measurements can be done with the same camera configuration used for the sky. Using the Moon needs some care, but it prevents from huge issues occurring when using point-like sources such as stars.

A set of pictures of the Moon is taken (overnight) with the Moon high over the horizon and with the sky as clear as possible. The exposure time $\Delta t$ has to be set in such a way that no 


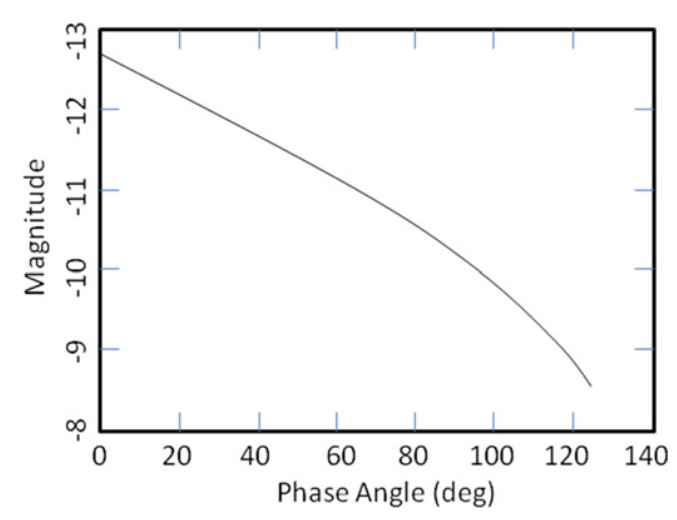

Figure 4. Lunar magnitude $m_{0}$ as a function of the phase angle $\varphi$ (adapted from [23]).

pixel saturates. Figure 3 shows one of the pictures used to calibrate, taken approximately $24 \mathrm{~h}$ from the full moon, $62^{\circ}$ over the horizon.

Up to a couple of days from the full moon the results are acceptable, while for larger phase angles the frequency histogram presents a non-negligible tail in the low channel region that makes it difficult to distinguish between the contribution from the faint regions of the Moon and the background light.

By means of equations (10a) and (11), the tone distribution shown in figure 3 can be converted to the corresponding relative radiance distribution $A(b)$, and the average relative radiance of the Moon finally converted into a tone through equation (11). In our case it corresponds to tone $b=135.7$.

The amount of light coming from the Moon depends on both its phase and its position in the sky, the latter determining the amount of air extinction. The sunlight reflected towards the Earth is not given just by the geometrical fraction of the illuminated area, because the shadows cast on the Moon by its mountains play an important role. The magnitude of the Moon as a function of the phase angle is shown in figure 4 (see for example [25]). Under the conditions of figure $3(\mathrm{a})$, namely for a phase angle $\varphi=12.6^{\circ}$, this curve gives $m_{0}=-12.3$. This is the magnitude of the Moon under ideal atmospheric conditions.

The atmospheric conditions that will give the magnitudes plotted in figure 4 are extremely rare. To correct for the effective atmospheric extinction, different approaches can be used [26-28]. The intensity of light impinging on the ground, $I$, for a source of intensity $I_{0}$ outside the atmosphere at a zenith angle $\theta$ can be obtained through the Beer-LambertBouguer extinction law:

$$
I=I_{0} e^{-k X}
$$

where $\mathrm{X}$ is the mass of the air, which can be obtained from the Rozember relation [26, 27]:

$$
X=(\cos \theta+0.025 \exp (-11 \cos \theta))^{-1}
$$

The parameter $k$ accounts for the atmosphere extinction. It is tabulated in the literature for different seasons, air conditions, zenith angle $\theta$ and altitude. In the case of figure 3 (winter time, sea level and zenith angle $\theta=62^{\circ}$ ) one gets $k=0.40$, and the Rozemberg relation gives an extinction factor $I / I_{0}=0.46$. The best atmospheric conditions give $k=0.11$, so in this ideal case the atmosphere would have an extinction factor of 0.84 . The ratio of these two values, 0.54 , provides the ratio between the amount of light impinging on the ground when the picture in figure 3 was taken and the amount of light that the Moon casts on the ground 
under the ideal atmospheric conditions considered in figure 4. The magnitude of the Moon in figure 3 can be evaluated from the value reported above, $m_{0}=-12.3$ :

$$
m_{\mathrm{M}}=m_{0}-2.5 \log _{10} 0.54=-11.6 .
$$

Since the Moon is an extended source, it is straightforward to refer to the specific magnitude, $m_{\mathrm{M}}$, expressed in magnitude $\operatorname{arcmin}^{-2}$. The solid angle $\Omega_{\mathrm{M}}$ subtended by the lunar disk can be evaluated on the basis of the apparent lunar radius when the picture was taken, in this case being approximately $\rho=0.28^{\circ}$ (the apparent radius has non-negligible changes; see [21]). The specific magnitude of the Moon is then:

$m_{\mathrm{M}}=-2.5 \log _{10}\left(I_{\mathrm{M}} /\left(I^{*} \Omega_{\mathrm{M}}\right)\right]=m_{\mathrm{M}}-2.5 \log _{10}\left(1 /\left(p r^{2}\right)\right]=-4.26 \operatorname{arcmin}^{-2}$.

From a solid angle of $1 \operatorname{arcmin}^{2}$ the Moon delivers an intensity which is that of Vega, $\mathrm{I}^{*}$, times 10 to the power $-m_{\mathrm{M}} / 2.5$ :

$$
r=6.07 \times 10^{-7} \mathrm{~W} \mathrm{~m}^{-2} \operatorname{arcmin}^{-2} .
$$

This is the reference radiance to be associated with the average tone $b=136.7$ and the relative radiance $A(b)$, and completes the absolute calibration of the response curve.

In conclusion, the radiance (or the specific magnitude) of the sky can be obtained by taking a picture under known conditions, measuring the average value of the tone distribution, $b^{\prime}$ and converting into the corresponding relative radiance, $A\left(b^{\prime}\right)$ through equation (11). The ratio between this value and the relative radiance obtained above for the Moon, $A\left(b^{\prime}\right)$, multiplied by the reference radiance $r$ in equation (16), provides the radiance of the sky.

\subsection{The daylight sky and the measure of $N_{A}$}

Once the camera is calibrated, data can be collected from the daylight sky to evaluate its radiance and therefore $N_{\mathrm{A}}$ as described above. Table 1 contains the results of some measurements at different altitudes and temperatures (all measurements were taken around midday). The first line contains theoretical results for pure air scattering at sea level. The values for $N_{\mathrm{A}}$ have been obtained from equation (5), including the observer altitude $z_{\mathrm{obs}}$, so that:

$$
N_{A}=\frac{B_{0}}{B_{C}} \frac{2}{3 \pi} M k^{4} \frac{\left(n_{0}-1\right)^{2}}{\rho_{0}} z_{0} \exp \left(-\frac{z_{\mathrm{obs}}}{z_{0}}\right) \frac{\Omega}{4 \pi} .
$$

The main source of errors should be the amount of suspended aerosols. By inspection of equation (17) this leads to a decrease of the expected $N_{\mathrm{A}}$, as occurs here. This means that from a large number of measurements we can discard the larger radiance values, which will be expected to be affected by non-molecular scattering (this selection must be done on data normalized for the altitude). In our case, for example, by using only the measurements in a very dry atmosphere from 2500 meters, one would estimate a value $N_{\mathrm{A}}=4.5 \times 10^{23} \mathrm{gmol}^{-1}$, which is not far from the expected value.

\section{Discussion and conclusions}

This method has been successfully proposed to undergraduate students several times, and always proved to be adequate. Different cameras have been used as well as different reference objects adopted for the calibration of the response curve (section 6.2). The history of 
Table 1. Experimental results obtained under different conditions.

\begin{tabular}{lccl}
\hline $\begin{array}{l}\text { Radiance } \\
\left(10^{-6} \mathrm{Wm}^{-2} \mathrm{arcmin}^{-2}\right)\end{array}$ & $\begin{array}{c}N_{\mathrm{A}}\left(10^{23}\right. \\
\left.\mathrm{gmol}^{-1}\right)\end{array}$ & $\begin{array}{c}\text { Altitude (meters } \\
\text { above sea level) }\end{array}$ & Comments \\
\hline 0.283 & 6.022 & 0 & Theory for pure air \\
0.222 & 5.58 & 2500 & Very clear sky-February $\left(-10^{\circ} \mathrm{C}\right)$ \\
0.265 & 4.66 & 2500 & Very clear sky-February $\left(-10^{\circ} \mathrm{C}\right)$ \\
0.348 & 3.54 & 2500 & Very clear sky-February $\left(-10^{\circ} \mathrm{C}\right)$ \\
0.350 & 3.56 & 2500 & Very clear sky-February $\left(-10^{\circ} \mathrm{C}\right)$ \\
0.50 & 2.71 & 2000 & Clear sky-August $\left(20{ }^{\circ} \mathrm{C}\right)$ \\
0.52 & 2.60 & 2000 & Clear sky-August $\left(20^{\circ} \mathrm{C}\right)$ \\
0.59 & 2.30 & 2000 & Clear sky-August $\left(20{ }^{\circ} \mathrm{C}\right)$ \\
1.23 & 1.06 & 2350 & Clear sky, humid-August $\left(20^{\circ} \mathrm{C}\right)$ \\
0.97 & 1.34 & 2350 & Clear sky, humid-August $\left(20{ }^{\circ} \mathrm{C}\right)$ \\
0.93 & 1.40 & 2350 & Clear sky, humid-August $\left(20{ }^{\circ} \mathrm{C}\right)$ \\
1.04 & 1.19 & 2800 & Clear sky, humid-August $\left(20^{\circ} \mathrm{C}\right)$ \\
0.97 & 1.27 & 2800 & Clear sky, humid-August $\left(20^{\circ} \mathrm{C}\right)$ \\
1.20 & 1.23 & 1200 & Clear sky-August $\left(25^{\circ} \mathrm{C}\right)$ \\
1.9 & 0.88 & 122 & Clear sky from town-May $\left(25^{\circ} \mathrm{C}\right)$ \\
3.0 & 0.55 & 122 & Clear sky from town-May $\left(25^{\circ} \mathrm{C}\right)$ \\
3.3 & 0.51 & 122 & Clear sky from town-May $\left(25^{\circ} \mathrm{C}\right)$ \\
\hline
\end{tabular}

questions about the daylight sky is a very instructive pathway for students, since it relates atoms, light, atmosphere, and several other common concepts that can be mixed in a quantitative, robust framework which is ultimately related to the proof of atomic theory by famous scientists at the beginning of the twentieth century. The absolute calibration of the camera response is good practice for learning how to calibrate a device and, once completed, students are able to perform measurements at will and to obtain results independently.

Care has to be taken to meet the atmospheric conditions for obtaining precise results. Nevertheless, an order-of-magnitude estimate is often possible, except for conditions that are clearly inconvenient. The best results can be obtained during winter and from high altitudes, when it is easier to find very dry and unpolluted air. Finally, this method has also been successfully proposed to undergraduate students for using the light from the sky to monitor air pollution.

\section{Acknowledgments}

The author gratefully acknowledges Davide Cenadelli for useful discussions and the anonymous referees for useful suggestions.

\section{References}

[1] Smoluchowski M 1908 Ann. Phys. 25 205-26

[2] Einstein A 1910 Ann. Phys. 33 1275-98

[3] Bohren C F 2003 Atmospheric Optics (New York: VCH)

[4] Pesic P 2005 Sky in A Bottle (Cambridge, MA: MIT Press)

[5] Tyndall J 1869 Phil. Mag. 37384

[6] Lord R 1899 Phil. Mag. 14377

[7] Lord R 1871 Phil. Mag. 14112 
[8] Pesic P P 2005 Eur. J. Phys. 26 183-7

[9] Mandel'stam L J 1907 Ann. Phys. 23626

[10] Landau L D and Lifsits E M 1986 Electrodynamics of Continuous Media (Moscow: MIR) par. 120

[11] Jackson J D 2000 Classical Electrodynamics (New York: Wiley) pp 425-6

[12] Crawford F S 1968 Waves: Berkeley Physics Course 3 (New York: Wiley) appendix A

[13] Marshall J and Plumb R A R A 2007 Atmosphere, Ocean and Climate Dynamics: An Introductory Text (New York: Academic)

[14] Jackson J D 2000 Classical Electrodynamics (New York: Wiley) p 155

[15] van de Hulst H C 1981 Light Scattering by Small Particles (New York: Dover) pp 36-9

[16] Wheeler J A and Feynman R P 1945 Rev. Mod. Phys. 17 157-81

[17] Lide D R (ed) 2004 Handbook of Chemistry and Physics (Boca Raton, FL: CRC Press)

[18] Kaufman V and Ward J F 1967 Appl. Opt. 643

[19] Meier R R 1991 Space Sci. Rev. 581

[20] King L V 1923 Proc. R. Soc. A 104333

[21] http://aa.usno.navy.mil/data/docs/diskmap.html

[22] Bjorn L O (ed) 2002 Photobiology. The Science of Light and Life (Dordrecht: Kluwer) p 43

[23] Thuillier G et al 2003 Sol. Phys. 214 1-22

[24] Kumar T S S, Jacob N M, Kurian C P and Shama K 2014 Int. J. Eng. Res. Technol. 3758

[25] Kieffer H H and Stone T C 2005 Astron. J. 129 2887-901

See also Luciuk M 2010 http://asterism.org/tutorials/tut26-1.htm

[26] Rozemberg G V 1966 Twilight: A Study in Atmospheric Optics (New York: Plenum)

[27] Green D W E 1992 Int. Comet Q. 14 55-9

[28] Young A T and Irvine W M 1967 Astron. J. 72 945-50 